Discussion Paper Series A No.474

\title{
The Behavior of Solutions to Bargaining Problems on the Basis of Solidarity
}

\author{
Yongsheng $\mathrm{Xu}$ \\ (Department of Economics, Andrew Young School of Policy Studies, \\ Georgia State University) \\ and \\ Naoki Yoshihara \\ (The Institute of Economic Research, Hitotsubashi University)
}

J anuary 2006

The Institute of Economic Research Hitotsubashi University

Kunitachi, Tokyo, 186-8603 J apan 


\title{
The Behavior of Solutions to Bargaining Problems on the Basis of Solidarity*
}

\author{
Yongsheng $\mathrm{Xu}$ \\ Department of Economics \\ Andrew Young School of Policy Studies \\ Georgia State University \\ Atlanta, GA 30303, U.S. A. \\ Email: yxu3@gsu.edu \\ and \\ Naoki Yoshihara \\ Institute of Economic Research \\ Hitotsubashi University \\ 2-4 Naka, Kunitachi \\ Tokyo, Japan 186-8603 \\ Email: yosihara@ier.hit-u.ac.jp
}

This version: December 2005

* We are grateful to William Thomson for comments on an earlier draft of the paper. 


\section{Introduction}

A persistent question asked in axiomatic bargaining problems is the following: when bargaining problems change from $A$ to $B$, how should solutions to them respond? Many of the axioms in the axiomatic bargaining literature are of this type. Among them, the most prominent ones are perhaps Nash's IIA (1950) and Kalai's Monotonicity (1977). Though they have been used mainly for understanding and characterizing different solutions to bargaining problems, a moment's reflection may convince us that they all have one thing in common: they are types of a broad class of axioms that may be called solidarity. The main idea underlying a solidarity-type axiom is that, when bargaining problems change from $A$ to $B$, the "utility gains" by any two players should not be in opposite directions: if one player gains from moving $A$ to $B$, then no player should become worse off from such a move; and if one player loses from moving $A$ to $B$, then no player should gain from such a move. The solidarity-type axioms have been fruitfully studied in the literature on fair allocations (see, for example, Fleurbaey and Maniquet (1999)). In the standard bargaining model, on the other hand, there exists little such systematic studies on solutions to bargaining problems based explicitly on the idea of solidarity. ${ }^{1}$ In this paper, we offer such a systematic study of the axioms based on solidarity for standard bargaining problems. As a by-product, we present alternative characterizations of some well-known solutions. Our alternative characterizations will provide a further understanding of the behavior of solutions to bargaining problems in terms of solidarity.

\section{Basic Model}

The set of players is denoted by $N=\{1,2, \ldots, n\}$ where $n \geq 2$. We use $\mathbf{R}_{+}$to denote the set of all non-negative real numbers, while $\mathbf{R}_{+}^{n}$ is used to denote the $n$-fold Cartesian product of $\mathbf{R}_{+}$. For each $\mathbf{x}, \mathbf{y} \in \mathbb{R}_{+}^{n}$, we write $\mathbf{x} \geq \mathbf{y}$ as $\left[x_{i} \geq y_{i}\right.$ for all $\left.i \in N\right], \mathbf{x}>\mathbf{y}$ as $\left[x_{i} \geq y_{i}\right.$ for all $i \in N$ and $\left.\mathbf{x} \neq \mathbf{y}\right]$, and $\mathbf{x} \gg \mathbf{y}$ as $\left[x_{i}>y_{i}\right.$ for all $\left.i \in N\right]$.

Let $\pi$ be a permutation of $N$. For each $\mathbf{x}=\left(x_{i}\right)_{i \in N} \in \mathbb{R}_{+}^{n}$, let $\pi(\mathbf{x})=$ $\left(x_{\pi(i)}\right)_{i \in N}$. Let $\Pi$ be the set of all permutations of $N$.

\footnotetext{
${ }^{1}$ Yoshihara $(2003,2005)$ discusses axiomatic characterizations of bargaining solutions in terms of solidarity in a specific model of production economies.
} 
Let $\mathcal{B}$ be the set of all compact, convex, and comprehensive subsets of $\mathbb{R}_{+}^{n}$, each of which contains an interior point of $\mathbb{R}_{+}^{n}$. Elements in $\mathcal{B}$ are interpreted as normalized (bargaining) problems. For each $A \in \mathcal{B}$ and each $\pi \in \Pi$, let $\pi(A)=\{\pi(\mathbf{a}) \mid \mathbf{a} \in A\}$. For each $A \in \mathcal{B}, A$ is a symmetric problem if $A=\pi(A)$ for all $\pi \in \Pi$.

For each $A \in \mathcal{B}$ and each $i \in N$, let $m_{i}(A)=\max \left\{a_{i} \mid\left(a_{1}, \cdots, a_{i}, \cdots, a_{n}\right) \in\right.$ $A\}$. Therefore, $\mathbf{m}(A) \equiv\left(m_{i}(A)\right)_{i \in N}$ is the ideal point of $A$.

For each $\mathbf{x} \in \mathbb{R}_{+}^{n}$ and $\boldsymbol{\alpha} \in \mathbb{R}_{++}^{n}$, let $\boldsymbol{\alpha}(\mathbf{x}) \equiv\left(\alpha_{i} x_{i}\right)_{i \in N}$. Given $A \in \mathcal{B}$ and $\boldsymbol{\alpha} \in \mathbb{R}_{++}^{n}$, let $\boldsymbol{\alpha}(A) \equiv\left\{\boldsymbol{\alpha}(\mathbf{x}) \in \mathbb{R}_{+}^{n} \mid \mathbf{x} \in A\right\}$. For each $A$ in $\mathbb{R}_{+}^{n}$, we define the comprehensive hull of $A$ by

$$
\operatorname{comp} A \equiv\left\{\mathbf{z} \in \mathbb{R}_{+}^{n} \mid \exists \mathbf{x} \in A: \mathbf{z} \leq \mathbf{x}\right\} .
$$

Let the convex hull of $A$ be denoted by $\operatorname{con} A$.

A solution $F$ is a single-valued mapping from $\mathcal{B}$ to $\mathbb{R}_{+}^{n}$ such that for every problem $A \in \mathcal{B}, F(A) \in A$. For given $F(A) \in A$, let $F_{i}(A) \in \mathbb{R}_{+}$be its $i$-th component. The following three are well-known solutions.

Nash Solution $F^{N A}$ : For every $A \in \mathcal{B}, F^{N A}(A)=\arg \max _{\left(a_{1}, \ldots, a_{n}\right) \in A} \prod_{i \in N} a_{i}$.

Kalai-Smorodinsky Solution $F^{K S}:$ For every $A \in \mathcal{B}, \quad F^{K S}(A) \in A$ implies that: (1) there is no other $\mathbf{a} \in A$ such that $\mathbf{a} \gg F^{K S}(A)$; and (2) there exists $\gamma \in(0,1)$ such that $F^{K S}(A)=\gamma \cdot \mathbf{m}(A)$.

Egalitarian Solution $F^{E}:$ For every $A \in \mathcal{B}, F^{E}(A) \in A$ implies that: (1) there is no other $\mathbf{a} \in A$ such that $\mathbf{a} \gg F^{E}(A)$; and (2) $F_{i}^{E}(A)=F_{j}^{E}(A)$ for all $i, j \in N$.

\section{Axioms and their relations}

We group axioms into two categories: non-solidarity type and solidarity type. We consider first the non-solidarity type. They are fairly standard in the literature on convex problems (see, for example, Peters (1992) and Thomson (1994) for discussions).

Efficiency (E): For each $A \in \mathcal{B}$, there is no $\mathbf{x} \in A$ such that $\mathbf{x}>F(A)$.

Weak Efficiency (WE): For each $A \in \mathcal{B}$, there is no $\mathbf{x} \in A$ such that $\mathbf{x} \gg F(A)$. 
Symmetry (S): For each $A \in \mathcal{B}$, if $A$ is symmetric, then $F_{i}(A)=F_{j}(A)$ for all $i, j \in N$.

Scale Invariance (SI): For all $A, B \in \mathcal{B}$, and each $\boldsymbol{\alpha} \in \mathbb{R}_{++}^{n}$, if $B=\boldsymbol{\alpha}(A)$, then $F(B)=\boldsymbol{\alpha}(F(A))$.

Next, we turn to solidarity type axioms.

Solidarity (SOL): For all $A, B \in \mathcal{B}$, either $F(A) \gg F(B)$ or $F(B) \gg$ $F(A)$ or $F(A)=F(B)$.

Restricted Solidarity (RSOL): For all $A, B \in \mathcal{B}$ with $\mathbf{m}(A)=\mathbf{m}(B)$, either $F(A) \gg F(B)$ or $F(B) \gg F(A)$ or $F(A)=F(B)$.

Weak Solidarity (WSOL): For all $A, B \in \mathcal{B}$, either $F(A) \geq F(B)$ or $F(B) \geq F(A)$.

Contraction Independence (CI): For each $A, B \in \mathcal{B}$, if $A \subseteq B$ and $F(B)$ is efficient on $A$, then $F(B)=F(A)$.

Restricted Contraction Independence (RCI): For each $A, B \in \mathcal{B}$ such that $\mathbf{m}(A)=\mathbf{m}(B)$, if $A \subseteq B$ and $F(B)$ is efficient on $A$, then $F(B)=$ $F(A)$.

Expansion Independence (EI): For each $A, B \in \mathcal{B}$, if $A \subseteq B$ and $F(A)$ is efficient on $B$, then $F(B)=F(A)$.

Restricted Expansion Independence (REI): For each $A, B \in \mathcal{B}$ such that $\mathbf{m}(A)=\mathbf{m}(B)$, if $A \subseteq B$ and $F(A)$ is efficient on $B$, then $F(B)=$ $F(A)$.

(SOL) is the most general form of a solidarity-type axiom. It requires that, whenever bargaining problems change from $A$ to $B$, either $F(A) \gg$ $F(B)$ or $F(B) \gg F(A)$ or $F(A)=F(B)$, meaning that if one player gains (loses) as a result from the move, every player gains (loses) as well. (RSOL) is a restricted version of (SOL). (WSOL) conveys the same idea as (SOL) in a weak form in which, when bargaining problems change from $A$ to $B$, it should be the case in which no player's gain is at the expense of some other player's loss. It may be noted that these three axioms are new in the literature. (CI) 
and (RCI) each deal with situations where a bargaining problem $A$ shrinks to another problem $B$. (CI) requires that, when a problem $B$ shrinks to another problem $A$, if the solution to $B$ is efficient on $A$, then $F(B)$ should continue to be the solution to $A$. The solidarity idea embedded in the axiom is that, given that $F(B)$ is efficient on $A$, any movement away from $F(B)$ will make at least one player worse off, and as a consequence, to keep the spirit of solidarity, $F(B)$ should continue to be the solution to $A$. It may be noted that (CI) is formally slightly weaker than Nash's IIA (1950), and also that (RCI) is formally slightly weaker than Restricted IIA proposed by $\mathrm{Yu}$ (1973). On the other hand, (EI) and (REI) each deal with situations involving the expansion of bargaining problems from $A$ to $B$. For example, (EI) requires that, when a problem $A$ is enlarged to another problem $B$, if the solution $F(A)$ to $A$ is efficient on $B$, then $F(A)$ should continue to be the solution to the problem $B$. The idea is that, even though there is an enlargement of "opportunities" from $A$ to $B$, given that $F(A)$ is both efficient on $A$ and on $B$, and that $F(A)$ is already the solution to the original problem $A$, any movement away from $F(A)$ will hurt at least one player, and thus the solution to the enlarged problem $B$ should continue to be $F(A)$. This requirement is consistent with the solidarity idea embedded in the solution. The property can also be seen as stating a certain inertia of the choice process. (REI) is weaker than (EI) in that it restricts its applicability to situations where the ideal point remains unchanged.

The following proposition summarizes the logical relationships between and/or among the solidarity-type axioms discussed above. The proof is simple and we leave it to the reader.

Proposition 1. (i) (SOL) $\Rightarrow$ (RSOL) and (WSOL); (ii) (SOL) $+(\mathrm{WE}) \Rightarrow$ (CI), (RCI), (EI), and (REI); (iii) (RSOL) $+(\mathrm{WE}) \Rightarrow(\mathrm{RCI})$ and (REI); (iv) $(\mathrm{WSOL})+(\mathrm{E}) \Rightarrow(\mathrm{CI}) \Rightarrow(\mathrm{RCI}) ;(\mathrm{v})(\mathrm{WSOL})+(\mathrm{E}) \Rightarrow(\mathrm{EI}) \Rightarrow(\mathrm{REI})$.

\section{Results and Their Proofs}

This section presents our main results and their proofs follow.

Theorem 1: The following statements are equivalent: (1.i) $F=F^{E}$; (1.ii) $F$ satisfies (WE), (S), (CI) and (EI); (1.iii) $F$ satisfies (WE), (S), (SOL).

Proof. We first establish the equivalence of (1.i) and (1.ii). It can be checked 
that $F^{E}$ satisfies (WE), (S), (CI) and (EI). We now show that if $F$ satisfies (WE), (S), (CI) and (EI), then $F=F^{E}$.

Let $F$ be a solution satisfying (WE), (S), (CI) and (EI). By non-emptiness of $F$ and (WE), we need only to show the following

For each $A \in \mathcal{B}$, each $\mathbf{x}$ and a that are weakly efficient in $A$, if $\left[a_{i}=a_{j}\right.$ for all $\left.i, j \in N\right]$, but $\left[x_{i} \neq x_{j}\right.$ for some $\left.i, j \in N\right]$, then $\mathbf{x} \neq F(A)$.

Let $\mathbf{x}$ and $\mathbf{a}$ be such that both are weakly efficient on $A,\left[a_{i}=a_{j}\right.$ for all $i, j \in N]$, and $\left[x_{i} \neq x_{j}\right.$ for some $\left.i, j \in N\right]$. Suppose to the contrary that $\mathbf{x}=F(A)$. Consider $B \equiv \operatorname{comp}\{\mathbf{x}\}$. Note that $B \subseteq A$. By $(\mathrm{CI}), \mathbf{x}=F(B)$.

Consider the set con $\left[\cup_{\pi \in \Pi} \pi(B)\right]$, and denote it by $C$. By construction, $C$ is a symmetric convex set having $C \supseteq B$. By the construction of $B$ and $C, \mathbf{x}$ is efficient on $C$. Therefore, noting that $\mathbf{x}=F(B), B \subseteq C$ and $\mathbf{x}$ is efficient on $C, \mathbf{x}=F(C)$ follows from (EI). Since $C$ is symmetric, by (WE) and $(\mathrm{S}), F(C)$ must be weakly efficient and be the equal utility point, which is a contradiction. Therefore, $\mathbf{x} \neq F(A)$. This proves (1.ii) implies (1.i), and thus the equivalence of (1.i) and (1.ii).

To complete the proof, we note that, $F^{E}$ satisfies (SOL), and that (SOL) and (WE) imply (CI) and (EI). $\diamond$

Theorem 2: The following statements are equivalent: $(2 . \mathrm{i}) F=F^{K S}$; (2.ii) $F$ satisfies (WE), (S), (SI), (RCI) and (REI); (2.iii) $F$ satisfies (WE), (S), (SI) and (RSOL).

Proof. The proof of Theorem 2 is similar to that of Theorem 1 by noting axiom (SI) and we leave it to the reader. $\diamond$

Our characterizations of the egalitarian and the KS solutions are new. Since we use variants of the solidarity-type axioms, our results offer a new perspective for these two solutions on the basis of solidarity. We also note that the Nash solution can be characterized by our (CI), together with (E), (S) and (SI). Since (CI) can be regarded as a solidarity-type axiom, the Nash solution has a certain sense of solidarity. Among the three solutions, clearly, the egalitarian solution fares the best in terms of solidarity since it can be characterized by using the strongest form of the solidarity-type axioms.

It can be checked that the axioms in each of our characterizations of the egalitarian solution are independent. The independence of the axioms (WE), (S), (SI) and (RSOL) in characterizing the K-S solution can be checked. For $\# N>2$, the axioms (WE), (S), (SI), (RCI) and (REI) are independent. If, 
however, $\# N=2$, then the $\mathrm{K}-\mathrm{S}$ solution is characterized by (WE), (S), (SI) and (REI). Thus, (RCI) is no longer indispensable to characterize the K-S solution in two-person problems.

\section{References}

1. Fleurbaey, M, and F. Maniquet (1999): "Fair allocation with unequal production skills: The solidarity approach to compensation,", Social Choice and Welfare 16, 569-583.

2. Kalai, E. (1977): "Proportional solutions to bargaining situations: interpersonal utility comparisons," Econometrica 45, 1623-1630.

3. Nash, J. F. (1950): "The bargaining problem," Econometrica, 18, 155162.

4. Peters, H. J. M. (1992): Axiomatic Bargaining Game Theory, Kluwer Academic Press.

5. Thomson, W. (1994): "Cooperative Models of Bargaining," in Handbook of Game Theory with Economic Applications, Aumann, R. J. and S. Hart (eds.), Elsevier.

6. Yoshihara, N. (2003): "Characterizations of Bargaining Solutions in Production Economies with Unequal Skills," Journal of Economic Theory 108, 256-285.

7. Yoshihara, N. (2005): "Solidarity and Cooperative Bargaining Solutions," forthcoming in Banach Center Publication series, Game Theory and Mathematical Economics.

8. Yu, P. L. (1973): "A class of solutions for group decision problems," Management Science 19, 936-946. 